Case Report

\title{
Remission of Hepatic Hydrothorax after OK-432 Pleurodesis
}

\author{
Taichiro Goto, MD, ${ }^{1}$ Yoshitaka Oyamada, MD, ${ }^{2}$ Reo Hamaguchi, MD, ${ }^{2}$ \\ Kumi Shimizu, MD, ${ }^{2}$ Masako Kubota, $\mathrm{MD},{ }^{2}$ Kumi Akanabe, MD, ${ }^{1}$ and Ryoichi Kato, $\mathrm{MD}^{1}$
}

\begin{abstract}
Hepatic hydrothorax in the absence of ascites is a rare complication of liver cirrhosis. A 71-year-old man with liver cirrhosis due to alcohol abuse was referred to our department because of massive pleural effusion on the right side. The properties of pleural effusion and clinical course led to a diagnosis of hepatic hydrothorax. Nonsurgical OK-432 pleurodesis resulted in a marked decrease of pleural effusion. After 2 months of follow-up, effusion was well-controlled. Patients with hepatic hydrothoraces have few options. OK-432 pleurodesis is relatively safe and may provide an effective alternative to peritoneovenous shunt, transjugular intrahepatic portosystemic shunt or surgical pleurodesis. It may also be a bridge toward liver transplantation in patients with few other options. Herein, we report a case of refractory hepatic hydrothorax successfully treated by nonsurgical OK-432 pleurodesis.
\end{abstract}

Key words: hepatic hydrothorax, liver cirrhosis, pleurodesis, OK-432

\section{Introduction}

Hepatic hydrothorax, caused by a flow of ascites into the pleural cavity, is a well-known complication of liver cirrhosis. ${ }^{1)}$ Patients with hepatic hydrothorax usually have advanced liver disease. The aim of therapy in such patients should be to relieve symptoms and prevent pulmonary complications until a liver transplantation can be performed. However, the optimal management remains unclear. Herein, we present a patient with recurrent pleural effusion secondary to hepatic cirrhosis who was treated with chest tube drainage and OK-432 pleurodesis, with ultimate resolution.

\footnotetext{
${ }^{1}$ Department of General Thoracic Surger, National Hospital Organization Tokyo Medical Center, Tokyo, Japan

${ }^{2}$ Department of Respiratory Medicine, National Hospital Organization Tokyo Medical Center, Tokyo, Japan
}

Received: November 6, 2009; Accepted: January 29, 2010 Corresponding author: Taichiro Goto, MD. (current affiliation) Department of Surgery, School of Medicine, Keio University, 35 Shinanomachi, Shinjuku-ku, Tokyo 160-8582, Japan

Email: taichiro@1997.jukuin.keio.ac.jp

(C)2011 The Editorial Committee of Annals of Thoracic and Cardiovascular Surgery. All rights reserved.

\section{Case Report}

A 71-year-old male with alcohol-related Child-Pugh class $\mathrm{C}$ liver cirrhosis was admitted to our department with dyspnea caused by massive right-sided pleural effusion in June 2009.

He was receiving outpatient treatment for alcoholic hepatitis, but did not recover from alcoholism, and continued drinking alcohol. He developed left-sided pyothorax in February 2009, was admitted to our department, and underwent pleural drainage and antibiotic therapy, which resulted in the healing of the pyothorax. A subsequent chest X-ray showed the full expansion of both lungs (Fig. 1A). In April 2009, he developed right pleural effusion. Thoracocentesis revealed that the pleural effusion was a sterile, nonmalignant transudate. Echocardiography showed no cardiac dysfunction, and other tests did not indicate cardiac or pulmonary disease as the cause of pleural effusion. The development of pleural effusion coincided with the worsening of liver function. Thus, he was diagnosed with hepatic hydrothorax from the clinical course as well and started on oral diuretic treatment and sodium restriction. Thereafter, he became and remained symptom-free for 2 months. 

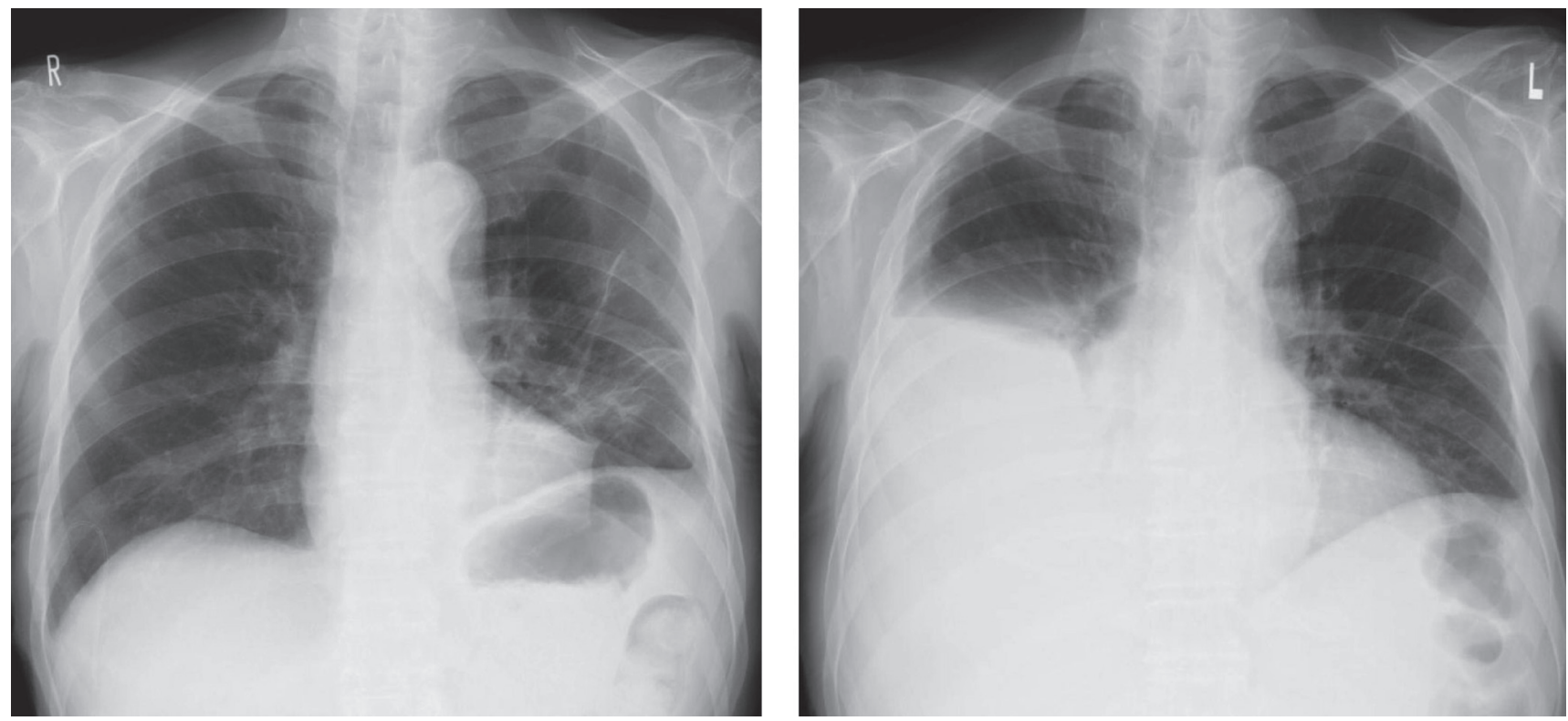

Fig. 1

A: Chest X-ray film (before admission) showing full expansion of the lungs without pleural effusion.

B: Chest X-ray film (after admission) showing right pleural effusion.

Physical examination revealed icteric sclera and bilateral lower extremity pitting edema, but no sign of ascites. The hemogram showed anemia and mild thrombocytopenia. Blood chemistry studies revealed hypoalubuminemia $(1.8 \mathrm{~g} / \mathrm{dl})$, and elevation of the total bilirubin $(1.69 \mathrm{mg} / \mathrm{dl})$, gamma-GTP $(223 \mathrm{U} / \mathrm{l})$ and $\mathrm{NH}_{3}(107$ $\mu \mathrm{g} / \mathrm{dl})$ levels. He showed a worsening of renal function with increases in BUN $(26.2 \mathrm{mg} / \mathrm{dl})$ and creatinine $(1.90$ $\mathrm{mg} / \mathrm{dl})$. The prothrombin time was slightly prolonged to $16.0 \mathrm{sec}$.

Chest radiography showed a large amount of right pleural effusion (Fig. 1B). Abdominal CT revealed marked liver cirrhosis and mild splenomegaly, but no ascites or focal hepatic lesion.

Because of the severity of the patient's dyspnea, chest tube was inserted to allow drainage of the effusion. The characteristics of the pleural effusion suggested its transudative nature. No bacteria or fungus was cultured from the effusion. Cytological examination of the pleural effusion showed no evidence of malignant disease.

The dyspnea improved significantly after chest tube insertion. The dose of diuretics was increased, and albumin was administered, but the amount of pleural effusion drained through the chest tube did not decrease. Since the patient did not respond to medical treatment, pleurodesis with $10 \mathrm{KE}$ of OK-432 (Picibanil, Chugai Pharmaceutical Co, Tokyo) was performed in July 2009. Over the next 2 weeks, the pleural effusion volume increased temporarily, and the chest-tube discharge was as high as $1000 \mathrm{ml} /$ day. Moreover, during the first several days after pleurodesis, the patient transiently developed worsening renal function and electrolyte abnormalities: BUN 50.7 mg/dl, Cr 3.04 mg/dl, Na 132 mEq/L, K 6.0 mEq/L. Fluid administration and electrolyte correction were performed, with symptomatic improvement. Three weeks after pleurodesis, the amount of pleural effusion drained markedly decreased to less than $50 \mathrm{ml}$ per day, allowing chest tube removal.

One month after tube removal, no pleural effusion was observed in the chest X-ray film (Fig. 2). No accumulation of ascites was noted after chest tube removal. The patient was discharged in September 2009 in a stable condition with a regimen of oral diuretics and is leading an uneventful life.

\section{Discussion}

Hydrothorax is the presence of more than $500 \mathrm{ml}$ of pleural effusion in a patient with liver cirrhosis who does not show pulmonary or cardiac diseases or malignancies.) Its onset, often sudden, is accompanied by severe respiratory distress. Pleural effusions complicate liver cirrhosis, occurring in approximately 0.4 to $12.2 \%$ of patients. ${ }^{2)}$ Strauss and Boyer reported incidences of $85.4 \%$ 


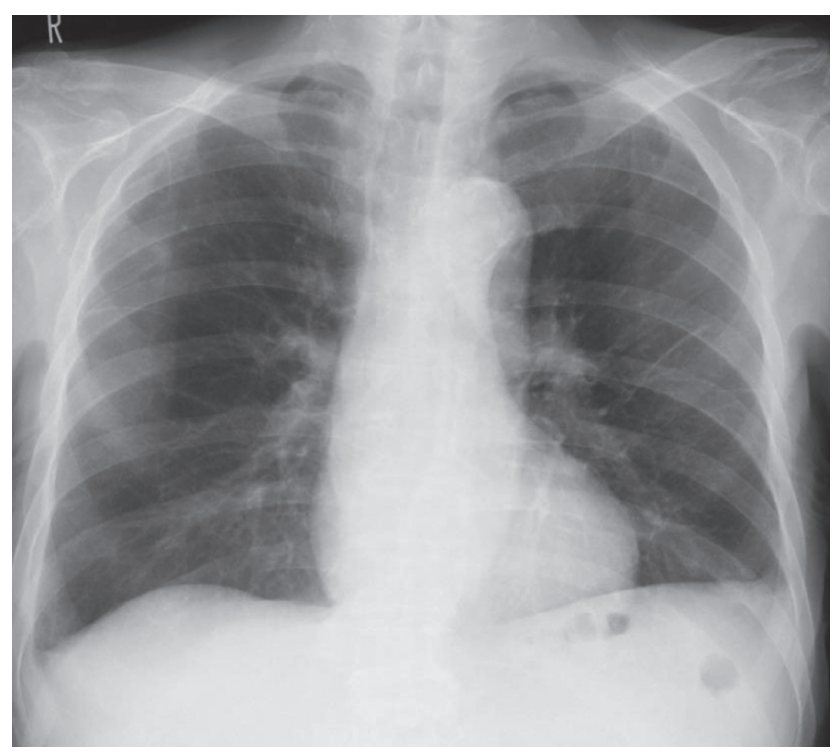

Fig. 2 Chest X-ray film (one month after chest tube removal) showing full expansion of the right lung without plural effusion.

right-sided, $12.5 \%$ left-sided, and 2\% bilateral hepatic hydrothoraces. ${ }^{1)}$ Recent studies have confirmed that small defects in the diaphragm allow the passage of ascitic fluid into the pleural space. ${ }^{3)}$ Hepatic hydrothorax occurs when the accumulation of ascites in the pleural cavity exceeds the absorptive capacity of the pleura. Although the source of leakage into the pleural cavity is unknown, it might be the left diaphragm because it is thinner and less muscular than the right, and it becomes even thinner with the application of high abdominal pressure. ${ }^{4)}$ Medical management includes sodium restriction and the aggressive use of diuretics. For those patients who do not respond medically, they instead undergo more invasive techniques, including serial thoracocentesis, chest tube placement, chemical or surgical pleurodesis, peritoneovenous shunt, and transjugular intrahepatic portosystemic shunt.

Hepatic hydrothorax in the absence of ascites is rare and may lead to diagnostic problems. ${ }^{5}$ Singer et al. demonstrated diaphragmatic defects upon the autopsy of patients with hepatic hydrothorax but without ascites, and proposed that hepatic hydrothorax without ascites might be caused by a rapid transdiaphragmatic movement of fluid from the peritoneal cavity to the pleural cavity prior to the formation of ascites. ${ }^{6}$

Recently, clinicians consider video assisted thoracoscopic surgery (VATS) pleurodesis with or without dia- phragmatic repairto be the technique of choice, ${ }^{7)}$ using the chemical agents: tetracycline and talc. ${ }^{78}$ However, patients with end-stage liver cirrhosis are most often poor surgical candidates, with high operative mortality rates.

OK-432 is an immune modulator purified from heatkilled Streptococcus pyogenes and is effective as a single agent in controlling malignant ascites and pleural effusion. Opinions as to the agent of choice for pleurodesis performed through tube thoracotomy vary, but because of inaccessibility to tetracycline and talc in Japan, Ok-432 has been widely used in pleurodesis for malignant pleural effusion, pneumothorax and chylothorax, with a high rate of effectiveness. ${ }^{9)}$ Renal dysfunction is a relative contraindication to the use of minocycline; therefore, it could not be used in this patient. In rare cases, talc may cause pneumonitis and adult respiratory distress syndrome; therefore, it should be carefully used. ${ }^{10)}$ To date, only a few Japanese and Taiwanese patients have undergone OK-432 pleurodesis for hepatic hydrothorax through a chest tube, with a high reported success rate. ${ }^{11,12)}$

While chemical pleurodesis by thoracoscopy is unquestionably the least aggressive technique for these fragile patients, the failure rate for all published series is more than $33 \%,{ }^{13}$ ) presumably because of the insufficient apposition of the pleural surfaces as a result of the rapid accumulation of pleural fluid. Chest tube drainage should be performed cautiously, especially in the presence of massive pleural effusion, because severe protein and electrolyte depletion with subsequent renal failure, impaired immunological function, and even death have been reported. ${ }^{14)}$ In the present case, too, pleurodesis worsened the renal function. However, by administering fluids and carefully monitoring serum electrolytes and renal function, we were able to place the chest tube and complete the procedure.

In this patient, the accumulation of ascitic fluid did not recur after pleurodesis. Ascites is present in patients with hepatic hydrothorax only if the formation of ascitic fluid exceeds its absorption by peritoneal lymphatics and transfer into the pleural space, but in this patient, the absorption capacity of the peritoneum was probably much higher than that of the pleura.

In conclusion, patients with end-stage liver cirrhosis and refractory hepatic hydrothorax have few therapeutic options. This case demonstrates that when surgical intervention is not suitable, OK-432 pleurodesis though a chest tube is a safe and effective alternative. 


\section{References}

1) Strauss RM, Boyer TD. Hepatic hydrothorax. Semin Liver Dis 1997; 17: 227-32.

2) Gordon FD, Anastopoulos HT, Crenshaw W, Gilchrist $\mathrm{B}$, McEniff N, et al. The successful treatment of symptomatic, refractory hepatic hydrothorax with transjugular intrahepatic portosystemic shunt. Hepatology 1997; 25: 1366-9.

3) Kirschner PA. Porous diaphragm syndromes. Chest Surg Clin N Am 1998; 8: 449-72.

4) Xiol X, Guardiola J. Hepatic hydrothorax. Curr Opin Pulm Med 1998; 4: 239-42.

5) Umino J, Tanaka E, Ichijoh T, Muraki T, Orii K, et al. Hepatic hydrothorax in the absence of ascites diagnosed by intraperitoneal spraying of indocyanine green. Intern Med 2004; 43: 283-8.

6) Singer JA, Kaplan MM, Katz RL. Cirrhotic pleural effusion in the absence of ascites. Gastroenterology 1977; 73: 575-7.

7) Assouad J, Barthes Fle P, Shaker W, Souilamas R, Riquet M. Recurrent pleural effusion complicating liver cirrhosis. Ann Thorac Surg 2003; 75: 986-9.
8) Falchuk KR, Jacoby I, Colucci WS, Rybak ME. Tetracycline-induced pleural symphysis for recurrent hydrothorax complicating cirrhosis. A new approach to treatment. Gastroenterology 1977; 72: 319-21.

9) Kishi K, Homma S, Sakamoto S, Kawabata M, Tsuboi E, et al. Efficacious pleurodesis with OK-432 and doxorubicin against malignant pleural effusions. Eur Respir J 2004; 24: 263-6.

10) Rinaldo JE, Owens GR, Rogers RM. Adult respiratory distress syndrome following intrapleural instillation of talc. J Thorac Cardiovasc Surg 1983; 85: 523-6.

11) Lin PY, Kuo PH, Yu CJ, Yang PC. Long-term remission of hepatic hydrothorax after OK-432 pleurodesis. J Thorac Cardiovasc Surg 2008; 136: 1367-9.

12) Saito R, Rai T, Saito H, Abe K, Takahashi A, et al. [Two cases of intractable hepatic hydrothorax successfully treated with nasal CPAP]. Nippon Shokakibyo Gakkai Zasshi 2006; 103: 1146-51. (in Japanese)

13) Ikard RW, Sawyers JL. Persistent hepatic hydrothorax after peritoneojugular shunt. Arch Surg 1980; 115: 1125-7.

14) Borchardt J, Smirnov A, Metchnik L, Malnick S. Treating hepatic hydrothorax. BMJ 2003; 326: 751-2. 